\title{
Crowd management using fuzzy logic and G.I.S.
}

\author{
N. P. Deshpande \& R. Gupta \\ Civil Engineering Department, BITS Pilani, India
}

\begin{abstract}
Our main objective is to design a computer based system to monitor the crowding system so as to avoid crowding disasters.

We have proposed a two-step mode. The first step is 'pre-disaster planning' including determination of sensitive locations and space management, evacuation paths using G.I.S. and management related arrangements. The second step is real time analysis of crowds to detect a possible emergency. It contains two modules, the first being a method to determine crowding situations and plan of action, and the second being the determination of the shortest evacuation path for the current area under surveillance. In the fuzzy inference system, used in determining crowding situation, crowd density is determined with the help of a number of pixels and shape of objects. For determining speed and cumulative displacement a new method is named 'determination of speed and displacement from images with help of object characterization'. The shortest path is determined with the help of G.I.S. and the overall crowding situation. We have considered two case studies:

1. Open air theatre: this particular case study was considered to get better understanding of general crowd movement patterns in the absence of crowd management systems and possible sensitive locations.

2. Auditorium: this case study was used to check the applicability of the overall project and to determine an evacuation path network. While applying this case study we checked for results such as accuracy and usability of components of the project such as crowd density determination, fuzzy inference system, Determination of speed and displacement etc., and overall usability.
\end{abstract}

Keywords: crowd management, crowd density, movement detection, image processing, use of fuzzy logic, G.I.S. application. 


\section{Introduction}

"Crowd - a large number of people gathered together with or without orderly arrangement" is the general definition of the word crowd. Crowding is our everyday experience. Especially in a country like India with such a large and alarmingly increasing population, we cannot escape the crowd wherever we may want to go, be it to a market, a sports venue, bus stops, railway stations or even a religious place. Although usually these gatherings take place without any serious troubles, occasionally, a combination of inadequate facilities, miscommunications and mismanagement results in crowding disasters and the mismanagement highlights it by resulting in an increased number of casualties. Consider, for an example, the recent crowding disaster of Chamunda Devi Temple in Jodhpur (Rajasthan state, India). The rumor, that a bomb has been planted in the temple, started the stampede but the effect of it was worsened to a greater extent due to steep and slippery slopes leading to the temple. 147 people died in this disaster.

Hence there is a need for some proper system to tackle such disasters. So that we can detect the dangerous crowding situation at the very beginning and we can control the flow of movement of people to avoid the disaster.

Nowadays different types of methods, ranging from the basic level (manual crowd management with barricades and crowd control forces), to the advance level (visual surveillance with automated equipment) are used for crowd management purposes. But the primary goal of all these systems, "To Enhance Public Safety", remains the same. However, each method has its own demerits. Through this paper we have tried to combine the pre-disaster planning, the real time surveillance and the manual control for the sake of better crowd management.

\section{Previous works}

\subsection{Crowd management theories (ref. to $[1,5,17])$}

Over the years many psychologists have proposed different kinds of theories about the crowd behavior during a crowding disaster. The following is an attempt to list a few of them:

\subsubsection{Panic model}

This model was proposed by a crowd psychologist named Gustav Le Bon in 1895. He proposed through this model that each individual is a different entity in the event of crowding disaster meaning everyone will have self preservation as the highest priority. The panic spreads like a wildfire in the crowd. The crowd does not have any cumulative identity. This self centered model was named as "Irrationalist theory". But later on this model was found to be incorrect due to observed behavior that was converse to this model. After this a "rationalist" theory was proposed which again was duly rejected. 


\subsubsection{Crowd behavior}

In this particular theory crowd behavior was suggested to be dependent mainly upon following three properties:

1. Internal Communication: lack of broader view.

2. Flight Response: reasonable action against perceived or real threat.

3. Craze: lure for a valuable object leading to selfish behavior.

Individual control is lost when the area available per person i.e. crowding density is lower than the human body area. This builds up intense crowd pressures resulting in fainting due to asphyxia (lack of oxygen in blood). As it is almost impossible to remove these people who have fainted they are crushed by the panicked crowd resulting in an increased number of deaths and injuries.

\subsection{Main aspects of crowd management: (ref. to [5])}

Crowd Management: The ability and execution of plans to organize or regulate a large number of people in an orderly arrangement. Aspects of crowd management:

\subsubsection{Managing information}

This part includes gathering initial information about the crowd, venue, training of employees, setting up surveillance systems, informing the crowd about emergency evacuation plans etc.

\subsubsection{Managing space}

- Attention to movement of people in building design projecting maximum occupancy level

- Dispersed and equally balanced ingress and exit points, e.g. positioning parking

- Simple alignment of pathways

- Back-up standby power

\subsubsection{Time based strategies}

These include the strategies to control rate of movement of people in the venue. Tactics include metering and early opening and delayed closing of the venue.

\subsubsection{Emergency response}

Sufficient space and equipment to handle the disasters should be available. Lives have been lost unnecessarily in large crowd incidents by the lack of simple equipment such as stretchers and oxygen cylinders. This response should be as quick as possible.

\section{Methodology and discussion}

\subsection{Overview}

A two-phase approach is proposed with

1. Pre-disaster Planning

2. Real time analysis. 
If this system is being set up for the first time in a particular venue, it will require four steps:
a) Acquiring basic information
b) Forming complete evacuation network
c) Training the system for obtaining crowd density
d) Based on images captured in real time determine crowding situation and best evacuation route in case of evacuation.

\subsection{Detailed description}

\subsubsection{Acquiring basic information}

This part is very crucial as by acquiring basic information about crowd as well as the venue, we can determine some preliminary parameters.

By acquiring information about expected crowd beforehand, we can know the approximate number of people who are going to gather at the venue, their possible behavior, age, mood etc. which helps us in determining a preliminary path for their entrance and exit as well. We can also determine if there is any such need of barricades etc.

By acquiring information about a venue, we can know about its area, dimensions and its map. All communication modes will also be checked during this process.

Every person associated with crowd management should be trained for all possible circumstances. All instructions and directives given must be clear to all employees.

\subsubsection{Evacuation path network}

Now that we know all the dimensions of the venue and also the positioning of barricades and any other obstacles, we know the complete path network of the venue. At this position the sensitive locations are determined. Sensitive locations are the locations where the possibility of crowding is higher. For better understanding of determination of sensitive locations, a case study of "Open air Theatre" was chosen. This venue was observed while around 200 people gathered in it and the sensitive locations were determined.

\subsubsection{Determination of sensitive locations and formation of network}

The methodology used above is suitable only for small environments, whereas for larger venues, with more complex structures, we need the help of computer programs. We divide the available area in smaller counterparts and represent the centre of each smaller area with a point. Now different checks, such as for exits, turns, change in crowd flow or crowding pattern etc, are made to determine the sensitive locations. These sensitive locations will be used for surveillance, as we will be keeping a constant vigil on these locations specifically. After determining the sensitive locations, a path network will be formed taking into consideration the available space for movement. 


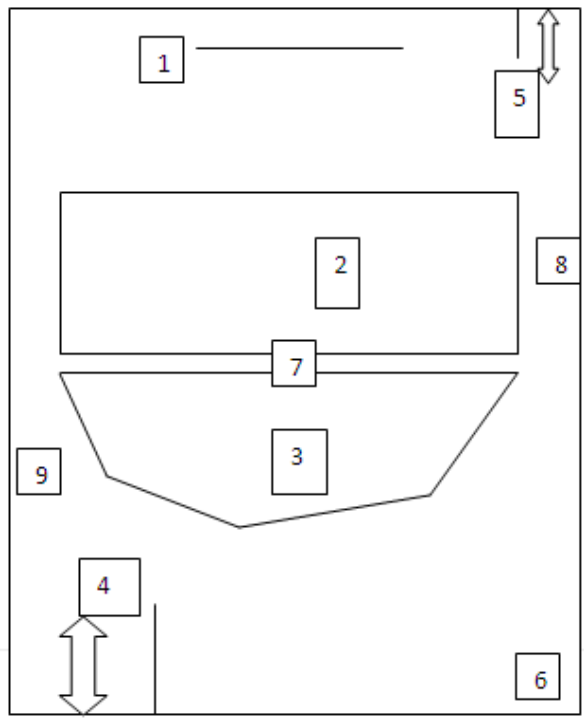

1. Screen

2. People (Sitting)

3. People (Standing)

4. Main exit(Sensitive)

5. Small exit(Sensitive)

6. Open air theatre

7. Sensitive location

8. Sensitive location

9. Sensitive location

Figure 1: $\quad$ Sensitive locations in case study.

\subsection{Real time analysis}

In this part our basic objectives will be to determine the crowding situation and to decide upon the action to be taken and in case of evacuation determining the best path of evacuation.

\subsubsection{Variables and their membership functions}

For determining a crowding situation and the action to be taken, we use Fuzzy logic. As the crowding depends on crowd density, space available, movement pattern and speed, we have considered 5 variables as:

- Local Crowd Density: this is the crowd density in the area which is currently under surveillance. As the locations where surveillance is to be done are fixed in sensitive location determination, we calculate the area under surveillance for each location. Now it remains to determine the number of people in that area, so that we can determine the crowd density.

$\mathrm{LCD}=$ area/number of people

- Global Crowd Density: this crowd density basically denotes the space occupancy of the complete venue. As we know the total number of people inside the venue, and the complete area which can be occupied, we determine the global crowd density as, $\mathrm{GCD}=$ Total area/Total no. of people in the venue.

- Time: this variable denotes a cumulative average speed of movement of people in the area we are considering. 
- Behavior: this variable denotes how the people are moving in that area, i.e. their cumulative average deflection from their line of movement.

- Future action: this variable indicates the tendency of movement of people from the area, i.e. if more people are moving in the area or moving out of it (change in the local crowd density).

Table 1: $\quad$ Variables and membership functions.

\begin{tabular}{|c|c|}
\hline Variable & Membership Function \\
\hline Local Crowd Density & Deadly, average, safe \\
\hline Global Crowd Density & Deadly, average, safe \\
\hline Time & Fast, average, slow \\
\hline Behavior & Angry, anxious, calm \\
\hline Future action & In, same, out \\
\hline
\end{tabular}

Now we apply a fuzzy inference system to these five variables with decision variable as the action to be taken in the current situation of crowding, i.e. to determine if it is safely crowded.

\subsubsection{Determination of crowd density}

This crowd management system requires all the required computations to be fast. In the case of determination of crowd density also the same principal holds. We need just the idea about the crowding situation of the place under consideration. For this purpose we follow the following process:

1. We capture consecutive images of the area under considerations.

2. Image Subtraction: in this process we get the difference in the two images. We get as a result an image which contains only the objects which are in image of interest but not in the original image. Each image captured in (1) is subtracted with the original image taken at the area of interest. As a result of subtraction (and a bit more image processing) we get only the people in the image.

3. Perimeter Detection: in this process just the outlines of the persons in the image is extracted with the help of changing pixel values.

4. Linear Correction: as the angle between camera and locations in image changes with the distance from camera, weight given to each pixel differs. This is taken into account while measuring number of pixels. 
5. Now we consider two aspects for estimating the number of persons in the image such as Number of pixels and shape of the objects. First the number of pixels in the edge detected image is calculated. To consider the shape of the objects, we label the connected object in the image and the total number of these connected objects is calculated. Each connected object indicates its shape.

6. Estimation of number: for this purpose we consider a large dataset of situations for each sensitive location. The steps above are applied to determine number of pixels and number of connected objects. Now for each such image, persons in the image are counted manually. This information for large set of images gives us the mean curves with help of which we can now estimate number of persons in the image when situation demands it and that too with fair accuracy.

By taking some more consecutive images of the area, we can apply the same method as above to determine change in crowd density with respect to time so as to know crowding tendency at the area under consideration.

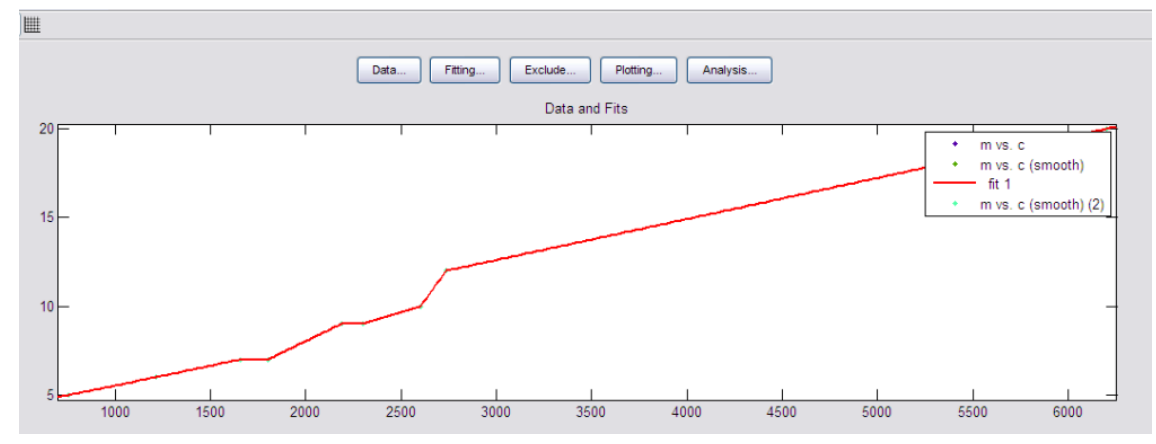

Figure 2: $\quad$ Crowd density estimation.

\subsubsection{Determination of speed and displacement of the crowd}

One alternative can be tracking each person to obtain the required fields, but it is clearly impractical for a larger crowd. Another method which seems ideal for the process can be as follows (ref. to [20]): By considering a sufficiently large DN value matrix around each pixel in both consecutive images and comparing those, we can detect the change in location of each person. With help of the same, we can calculate required properties. This solution does not exist in practicality due to the huge time taken.

We propose a different alternative; this method for detecting and determining motion of the object in the picture does not detect each individual and tract the motion of the person, instead this method deals with the shape and area of the objects in the image. First we follow steps 1 to 3 of crowd density determination.

- Label the connected objects in the image. Each connected object is a set of neighboring pixels with value 1 in a binary image. 
- Then we compare two successive images. We search for the same objects in these two images with help of their area. When we get area, to be equal for more than two images then we select the one which is closest (as the images are taken at successive interval resulting in lower displacement).

- Now we determine weighted centroids of each object in both images. With help of the centroid positions, the distance travelled by the object can be calculated by applying linear correction.

- Now that we have calculated both the distance travelled and the time taken, we can calculate their speed.

- We can also determine change in their movement pattern, i.e. their deflection from the original line of movement. The degree of deflection denotes the behavioral pattern of the crowd.

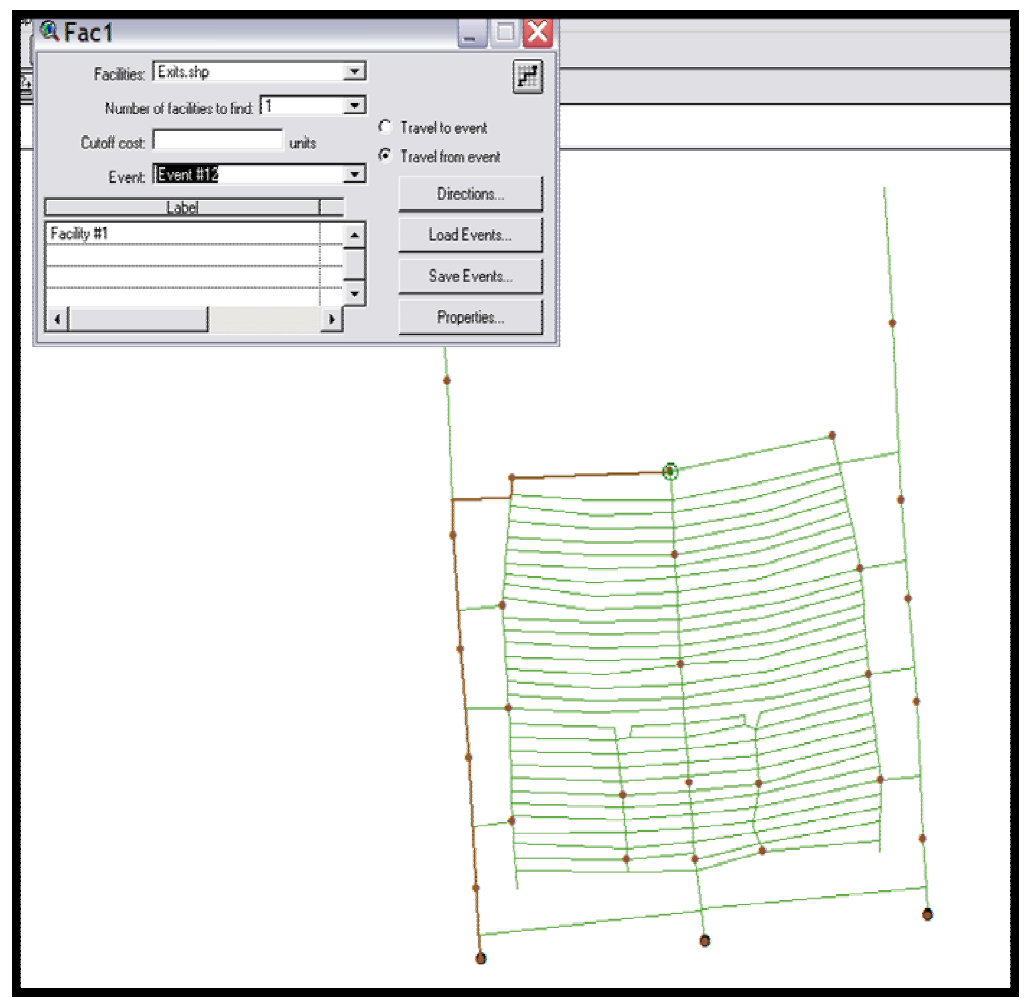

Figure 3: Closest evacuation path. 


\subsection{Determination of closest evacuation path}

Now the closest evacuation path is determined using the known complete evacuation network and the crowding status. We use "Finding Closest facility" (with facility being Exits) in network analyst for this purpose. We determine some new cost fields such as width and safety. Width denotes the width of the corresponding paths. Safety denotes the recent safety status at each sensitive location, i.e. each time the safety decision is calculated with process in previous sections that value is updated in respective position, based on which the weights are determined for each path. We can determine the final cost value for each path using multi criteria analysis and previously obtained values

\section{Conclusions}

The system proposed in the paper tries to deal with every aspect of crowd management. For any given venue, the system has the same basic structure at the start, but the system trains itself so as to cater the specific requirements of the venue. The steps used in the system are as follows - acquiring basic information, formation of evacuation network; training the system, calculation and decision. For greater usability we have tried to minimize the processing time. It results in the system being simple, fast, at the same time fairly accurate and hence, highly useful.

\section{Future scope}

The work for building as well as improving the complete system is in progress. In the near future we plan to work on mainly three areas, namely - user friendliness, increasing accuracy and lowering the setup cost. We would also like to check the system on larger datasets so as to improve it before it can be converted into a proper product. We plan to create separate software to incorporate all the required functions with usability and affordability.

\section{References}

[1] http://en.wikipedia.org/wiki/

[2] http://support.esri.com/index.cfm

[3] http://www.library.yale.edu

[4] http://www.cs.ucsb.edu

[5] http://www.crowddynamics.com

[6] http://www.thinklab.salford.ac.uk/

[7] http://www.iiit.ac.in/techreports/

[8] http://www.ukresilience.gov.uk/media/ukresilience/assets/

[9] http://www.sce.carleton.ca/faculty/chinneck/

[10] www.sagamorepub.com/powerpoint/FacilityDesign

[11] www.cs.cmu.edu/ jkh/video_games/group_behaviors.pdf

[12] http://cialab.ee.washington.edu 
[13] http://www.cs.berkeley.edu

[14] http://www.mil.ufl.edu

[15] http://math.haifa.ac.il

[16] http://partnersinpreparedness.com/conference/

[17] http://www.sussex.ac.uk/affiliates/panic/

[18] Klir George and Yuan Bo, 1995, Fuzzy sets and fuzzy logic theory and application

[19] Klir, J., and Foger, T. A. (1988), Fuzzy Sets, uncertainty and information, Prentice-Hall, Englewood Cliffs, N.J.

[20] Anthony C. Davies, Jia Hong Yin and Sergio A. Velastin, 1995, "Crowd Monitoring Using Image Processing”, IEE Electronic and Communications Engineering Journal, Vol 7, No. 1, pp.37-37

[21] Sylves, R.T., and Waugh,W.L. Jr. (1995). Disaster management in the U.S. and Canada; The policymaking, administration and analysis of emergency management, $2^{\text {nd }}$ Ed., Charles C. Thomas Publishers, Springfield, Ill. 